\title{
Characterization of Potential Pathogenic Bacteria Isolated in High-Risk Infectious Services at the University Hospital Center of Suru-Léré in Benin
}

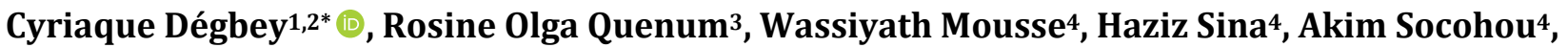 \\ Eric Hounsinou ${ }^{5}$, Lamine Baba-Moussa ${ }^{4}$, Honoré Bankolé ${ }^{3}$ \\ ${ }^{1}$ Regional Institute of Public Health, University of Abomey-Calavi, Ouidah, Benin \\ ${ }^{2}$ University Clinic of Hospital Hygiene, National Hospital and University Center Hubert Koutoukou MAGA, Cotonou, Benin \\ ${ }^{3}$ Polytechnic School of Abomey-Calavi, University of Abomey-Calavi, Abomey-Calavi, Benin \\ ${ }^{4}$ Laboratory of Biology and Molecular Typing in Microbiology, Abomey-Calavi, Benin \\ ${ }^{5}$ University Hospital Center of Suru-Léré, Cotonou, Benin \\ Email: *comlancy@yahoo.fr
}

How to cite this paper: Dégbey, C., Quenum, R.O., Mousse, W., Sina, H., Socohou, A., Hounsinou, E., Baba-Moussa, L. and Bankolé, H. (2021) Characterization of Potential Pathogenic Bacteria Isolated in High-Risk Infectious Services at the University Hospital Center of Suru-Léré in Benin. Advances in Microbiology, 11, 1-15. https://doi.org/10.4236/aim.2021.111001

Received: November 28, 2020

Accepted: January 9, 2021

Published: January 12, 2021

Copyright $\odot 2021$ by author(s) and Scientific Research Publishing Inc. This work is licensed under the Creative Commons Attribution International License (CC BY 4.0).

http://creativecommons.org/licenses/by/4.0/ (c) (i) Open Access

\begin{abstract}
Healthcare-associated infections (HCAIs) remain a major public health concern. The aim of this study was to characterize potential pathogenic bacteria isolated in high-risk infectious services at the University Hospital Center of Suru-Léré in Benin. A cross-sectional, descriptive study was carried out on 215 samples with 8 air samples, 20 hand samples and 187 samples of medical-technical material. Genes for resistance to $\beta$-lactam antibiotics (blaTEM, blaSHV, blaCTX-M and blaOXA-1) were sought. The data were processed and analyzed using EPI Info version 3.5.4 software. A total of 10 bacterial species were identified. Of the 319 bacterial strains identified, Staphylococcus saprophyticus, Staphylococcus aureus and Enterococcus faecalis were predominant at $20.06 \%, 17.87 \%$ and $11.28 \%$ respectively. Antibiotic susceptibility showed that the majority of bacterial strains were multidrug-resistant. Of the 4 resistance genes sought, only the blaTEM and blaSHV genes were found. The poor microbiological quality observed in high-risk infectious services could be a risk factor for healthcare-associated infections. It is therefore useful to implement preventive measures in order to prevent or reduce the risk of healthcare-associated infections.
\end{abstract}

\section{Keywords}

Bacteria, Infection, Risk Factors, Benin 


\section{Introduction}

Healthcare-associated infections (HCAIs) remain a major public health concern [1]. The multiple causes of HCAIs are related to systems, procedures and behavioral practices [2]. The consequences of their occurrence include prolonged hospital stays, excess costs, high antimicrobial resistance of microorganisms, and higher mortality or morbidity [3] [4]. To control HCAIs, many preventive measures have been implemented by the Hospital Infection Control Committees (NICCs). These main measures concern hand hygiene, isolation of sensitive individuals, monitoring of antibiotic use, and monitoring of the microbiological quality of air, water, soil and surfaces. Surveillance is one of the main components of the policy to reduce HCAIs [3] [5].

The risk of infection is everywhere in health care facilities. The healthcare environment contributes to the spread of pathogens, despite the progress made in health safety. Depending on the risk of infection to the patient, the premises of a health care facility can be classified into four zones. These are the low-risk zone, the medium-risk zone, the high-risk zone and the very high-risk zone. Thus, to manage and reduce the health problems that result, first step is to assess the level of contamination and identify germs in hospitals [6]. The fight against hospital infectious risks is therefore based on the implementation of rigorous medical hygiene practices and various preventive measures [7]. The application of interventions and strategies are likely to reduce the burden of morbidity and mortality due to HCAIs [2].

In Africa, the prevalence of HCAIs ranges from $2.5 \%$ to $14.8 \%$ [8]. Information on the pathogens responsible for these infections is provided by some data [9]. In Benin, the issue of HCAIs is not a new one. Strategies to combat this problem are included in the national hospital hygiene policy in the Republic of Benin [10]. Several studies have assessed the level of contamination of surfaces, medical and technical equipment, and premises in health care facilities [4] [11]. This study aimed to characterize potential pathogenic bacteria isolated in high-risk infectious services at the University Hospital Center of Suru-Léré (CHUZ-SL).

\section{Materials and Method}

\section{Study setting}

This study took place at the CHUZ-SL and the Laboratory of Biology and Molecular Typing in Microbiology (LBTMM). At the CHUZ-SL, the collection of samples, morphological characterizations, biochemical characterizations, and research of the antibiotic sensitivity of the isolated bacteria took place. In the LBTMM, we looked for virulence factors and resistance genes.

\section{Study population}

The study population consisted of surfaces (mattresses, sheets, door handles, over-blouses, hands of health personnel), air and medical-technical equipment (incubators, cradles, multiparameter apparatus, gallows, beds, operating table, surgical lamps, etc.). The departments under investigation were the neonatology, the recovery room, and the operating theatres. 


\section{Sampling}

The sample included 215 samples: 8 air samples, 20 hand samples, and 187 samples of medical-technical equipment.

\section{Data Collection}

According to the ISO/DIS 14698-1 standard, we use the swabbing technique to test for bacteria on technical medical equipment and the hands of health care personnel. To detect the presence of bacteria in the air, plate count agar (PCA), blood agar, Chapman agar, Bile Esculin Agar (BEA), Eosin Methylene Blue $(\mathrm{EMB})$ agar were placed for 30 minutes in the premises of the investigated departments.

\section{Material}

The different culture media used were Chapman Agar, PCA, Mueller Hinton, BEA, DNAse, EMB, Uriselect, Blood Agar, Mueller Hinton Broth, Brain Heart, Yeast Casamino-acid Pyruvate (YCP). During the study, we used various reagents such as Gram stain reagents, PCR reagent kit, Coomassie Blue, 10\% hydrochloric acid, hydrogen peroxide, oxidase strip, rabbit plasma, $\mathrm{API}^{\circledR} 20 \mathrm{E}$, and antibiotic discs. The equipment used consisted of refrigerators, freezers, ordinary microscope, mechanical balance, oven at $37^{\circ} \mathrm{C}$, autoclave, thermocycler, Electrophoresis Tank, ultra-violet trans-illuminator.

\section{Data Analysis}

\section{Culture and isolation of bacteria}

The swab technique was used to search for bacteria on medico-technical equipment and on the hands of health personnel. Sterile moistened swabs were passed in close parallel streaks, rotating them slightly. This gesture was repeated in the same area with streaks perpendicular to the horizontal streaks. The swabs returned to their protective cases were sent to the laboratory.

The agar plates placed for air sampling were incubated for 24 hours at $37^{\circ} \mathrm{C}$. The swabs were inoculated in $5 \mathrm{~mL}$ Mueller Hinton (MH) broth and then incubated for 24 hours at $37^{\circ} \mathrm{C}$. Bacterial growth resulted in the turbidity of the $\mathrm{MH}$ broth and the presence of colonies on the agar plates. A fresh state and Gram staining were performed on the cloudy broths. Each cloudy MH broth was then inoculated by the depletion isolation technique on agar plates such as Chapman, EMB, BEA, blood agar, Uriselect. The agar plates inoculated were incubated at $37^{\circ} \mathrm{C}$ for 24 hours.

\section{Identification of bacteria}

The study of morphological, cultural, and biochemical characteristics allowed the identification of isolated bacteria. After incubation, we performed Gram stain controls and the following biochemical tests: the search for coagulase, DNAse, catalase, oxidase, and the identification by the API 20E gallery [12]. We used the radial immunoprecipitation technique or Ouchternoly method to detect Panton-Valentine Leukocidin (LPV), a toxin produced by staphylococci [13] [14]. 


\section{Antibiogram}

Antibiotic susceptibility was studied using the $\mathrm{MH}$ agar diffusion method (Table 1). The MH agar is inoculated by flooding with a $0.5 \mathrm{McFarland}$ bacterial suspension. Antibiotic discs are deposited on the inoculated agar and dried. Incubation is done at $37^{\circ} \mathrm{C}$ in an oven for 24 hours. The interpretation was made according to the recommendations of the Antibiogram Committee of the French Society for Microbiology. Phenotypic detection of ESBL production was also performed [15] [16]. The antibiotics tested are grouped together in Table 1. These antibiotics were chosen according to the recommendations of the French Society of Microbiology.

\section{Search for resistance genes using molecular genetic markers}

This research was carried out using the Polymerase Chain Reaction (PCR) technique.

\section{Extraction of DNA}

The extraction of DNA from the bacterial strains was done according to the method adapted from Rasmussen and Morrisey [17]. The bacterial strains transplanted from $\mathrm{MH}$ agar were emulsified in $1 \mathrm{~mL}$ of $\mathrm{MH}$ broth. After incubation at $37^{\circ} \mathrm{C}$ for 18 hours, the resulting bacterial cultures were centrifuged at 12,000 rpm for 5 minutes. After the second wash, heating in a dry bath at $95^{\circ} \mathrm{C}$ for 20 minutes was done. The supernatant obtained after centrifugation was transferred to another Eppendorf tube. $500 \mu \mathrm{l}$ of $100 \%$ ethanol was added. After further centrifugation, the supernatant obtained was discarded. The DNA is collected in a mass at the bottom of the Eppendorf tubes. This DNA pellet was then dried at room temperature under the laminar flow hood.

Table 1. Different families of antibiotics according to the discs tested.

\begin{tabular}{|c|c|c|c|}
\hline & Groups & Antibiotics & Charge \\
\hline \multirow{8}{*}{$\beta$-LACTAMINS } & \multirow{4}{*}{ Penicillins } & Penicillin G $(\mathrm{P})$ & $1 \mu \mathrm{g}$ \\
\hline & & Ampicillin (AMP) & $10 \mu \mathrm{g}$ \\
\hline & & Amoxicillin (AML) & $25 \mu \mathrm{g}$ \\
\hline & & Amoxicillin + Clavulanic acid (AMC) & $30 \mu \mathrm{g}$ \\
\hline & Carbapenems & Ertapenem (ETP) & $10 \mu \mathrm{g}$ \\
\hline & \multirow{2}{*}{ Cephalosporins } & Cefoxitin (FOX) & $30 \mu \mathrm{g}$ \\
\hline & & Cefotaxime (CTX) & $30 \mu \mathrm{g}$ \\
\hline & Monobactams & Aztreonam (ATM) & $30 \mu \mathrm{g}$ \\
\hline \multirow{2}{*}{\multicolumn{2}{|c|}{ AMINOSIDES }} & Gentamycin (GN) & $10 \mu \mathrm{g} / 500 \mu \mathrm{g}$ \\
\hline & & Netilmicin (NET) & $10 \mu \mathrm{g}$ \\
\hline CYCL & INES & Tétracycline (TE) & $30 \mu \mathrm{g}$ \\
\hline \multirow{2}{*}{ M.L.S } & Macrolides & Erythromycin (E) & $15 \mu \mathrm{g}$ \\
\hline & Lincosamides & Lyncomycin (MY) & $15 \mu \mathrm{g}$ \\
\hline \multirow{2}{*}{\multicolumn{2}{|c|}{ QUINOLONES }} & Norfloxacin (NOR) & $10 \mu \mathrm{g}$ \\
\hline & & Ciprofloxacin (CIP) & $5 \mu g$ \\
\hline
\end{tabular}




\section{DNA Amplification}

The PCR reaction was done in thermal cyclers. Primers of the $16 \mathrm{~S}-23 \mathrm{~S}$ gene were used to identify strains of staphylococci. The primers blaTEM, blaSHV, blaCTX-M, and blaOXA-1 were used to search for resistance genes of Gramnegative bacterial strains. The reaction volume for the search for the different genes was $25 \mu \mathrm{l}$. It contained $7.5 \mu \mathrm{l}$ of bacterial DNA; $2.5 \mu \mathrm{l}$ of each primer ( $\mathrm{F}$ and R) and $12.5 \mu$ of Master Mix 2X. The program used for the amplification of the $16 \mathrm{~S}-23 \mathrm{~S}$ gene was as follows: initial denaturation at $94^{\circ} \mathrm{C}$ for 2 minutes followed by 25 cycles (denaturation at $94^{\circ} \mathrm{C}$ for 1 minute, hybridization at $47^{\circ} \mathrm{C}$ for 7 minutes, elongation at $72^{\circ} \mathrm{C}$ for 2 minutes) followed by a final elongation at $72^{\circ} \mathrm{C}$ for 10 minutes. The program used for the amplification of the blaSHV, blaCTX-M and blaOXA-1 genes was an initial denaturation at $94^{\circ} \mathrm{C}$ for $10 \mathrm{mi}-$ nutes, 30 cycles (denaturation at $94^{\circ} \mathrm{C}$ for 40 seconds, hybridization at $60^{\circ} \mathrm{C}$ for 40 seconds, elongation at $72^{\circ} \mathrm{C}$ for 1 minute) and a final elongation at $72^{\circ} \mathrm{C}$ for 7 minutes. The program used for the amplification of the blaTEM gene was as follows: initial denaturation at $94^{\circ} \mathrm{C}$ for 5 minutes; 30 cycles (denaturation at $94^{\circ} \mathrm{C}$ for 30 seconds, hybridization at $52^{\circ} \mathrm{C}$ for 30 seconds, elongation at $72^{\circ} \mathrm{C}$ for 1 minute) and final elongation at $72^{\circ} \mathrm{C}$ for 10 minutes [18]. The sequences and fragments of the 16S - 23S, blaTEM, blaSHV, blaCTX-M and blaOXA-1 primers used are presented in Table 2.

\section{Agarose gel electrophoresis}

The verification of the presence of DNA was done by agarose gel electrophoresis. The size of the PCR products was determined by comparison with a DNA ladder of the molecular weight marker. This molecular weight marker contains DNA fragments of known size. The $1.2 \%$ agarose gel was prepared by dissolving $1.2 \mathrm{~g}$ of the agarose gel in $100 \mathrm{ml}$ of TBE $1 \mathrm{X}$ (Tri Base EDTA). The prepared solution is boiled. $1 \mu \mathrm{l}$ of BET (Ethidium Bromide) was added after slight cooling. The BET intercalates between the nitrogen bases. It emits ultraviolet fluorescent

Table 2. Different resistance genes of interest.

\begin{tabular}{|c|c|c|c|c|}
\hline & Primers & Primer sequences $\left(5^{\prime} \rightarrow 3^{\prime}\right)$ & Genome size & Reference \\
\hline \multirow{2}{*}{ blaTEM } & OT-F & 5'ATTGGGTGCACGAGTGGGTTAC-3' & \multirow{2}{*}{467} & \multirow{2}{*}[18]{} \\
\hline & OT-R & 5'-ATAATTGTTGCCGGGAAGCTAG-3' & & \\
\hline \multirow{2}{*}{ blaSHV } & SHV-F & 5'AGCCGCTTGAGCAAATTAAAC-3' & \multirow[b]{2}{*}{713} & \multirow[b]{2}{*}{ [18] } \\
\hline & SHV-R & 5'-ATCCCGCAGATAAATCACCAC-3' & & \\
\hline \multirow{2}{*}{ blaCTX-M } & CTX-F & 5'-TTAGGAARTGTGCCGCTGYA-3' & \multirow{2}{*}{688} & \multirow{2}{*}{ [18] } \\
\hline & CTX-R & 5'-CGATATCGTTGGTGGTRCCAT-3' & & \\
\hline \multirow[b]{2}{*}{ blaOXA-1 } & OXA-1-F & 5'-GGCACCAGATTCAACTTTCAAG-3' & \multirow[b]{2}{*}{564} & \multirow[b]{2}{*}{ [18] } \\
\hline & OXA-1-R & 5'-GACCCCAAGTTTCCTGTAAGTG-3' & & \\
\hline \multirow{2}{*}{$16 S-23 S$} & G-1 & 5'-GAAGTCGTAACAAGG-3' & \multirow{2}{*}{$237-437$} & \multirow{2}{*}{ [18] } \\
\hline & L-1 & 5'-CAAGGCATCCACCGT-3' & & \\
\hline
\end{tabular}


light. The gel containing the BET was poured into the vessel. The fine combs are placed in the warm gel. They are removed after solidification of the gel. They leave holes in the gel called wells. This gel is then deposited in the migration basin. It was deposited respectively in the wells: $8 \mu \mathrm{l}$ of the 100 base pair molecular weight marker (Biolabs), a negative control, a positive control, and PCR products (or dissolved DNA pellets). Migration on 1.2\% agarose gel was performed in TBE $1 \mathrm{X}$ buffer, under a constant voltage of $100 \mathrm{~V}$ for 30 minutes. The agarose gel was visualized using an ultra-violet transilluminator. Wherever fluorescence is emitted, BET can be said to be present. If there is BET, it means that there are nitrogenous bases. Nitrogenous bases signal the presence of DNA in PCR products or DNA pellets.

\section{Statistical analysis}

The data were processed and analyzed using EPI Info version 7 and MiniTab 17 software.

\section{Results}

Bacterial species present in medical-technical equipment, hands of personnel and the air

About $81 \%$ of the bacterial strains were isolated from medical-technical equipment. A total of 10 bacterial species were identified (Acinetobacter baumannii, Enterobacter cloacae, Enterococcus faecalis, Klebsiella pneumoniae, Proteus vulgaris, Pseudomonas aeruginosa, Staphylococcus aureus, Staphylococcus epidermidis, Staphylococcus saprophyticus, Streptococcus agalactiae). The most frequently isolated were Staphylococcus saprophyticus (20.06\%), Staphylococcus aureus (17.87\%), Enterococcus faecalis (11.28\%) and Acinetobacter baumannii (10.66\%). Among the 10 bacterial species identified, 3 were found at all sampling sites at the same time. In neonatology, the predominant bacterial species was Staphylococcus aureus (23.91\%). It was followed by Enterococcus faecalis (13.04\%) and Pseudomonas aeruginosa (11.96\%). In the recovery room, Enterococcus faecalis (20.27\%) and Staphylococcus saprophyticus (18.92\%) were the most isolated bacterial species. These two bacterial species were found at all sampling sites. In the operating theatres, Staphylococcus saprophyticus was the most isolated (26.79\%). It was found at all three sites.

\section{Resistance profile of identified bacteria to antibiotics}

Gram-positive bacterial resistance to the families of antibiotics tested

The strains of Enterococcus faecalis had 7 different antibiotic resistance profiles with multidrug-resistance observed in $73.08 \%$ of cases. Most strains of Staphylococcus aureus (89.66\%) were multidrug-resistant (MDR) with 9 different resistance patterns observed. The vast majority (92.30\%) of Staphylococcus epidermidis strains were MDR with 6 different resistance patterns. Strains of Staphylococcus saprophyticus had 9 different resistance profiles with $85.71 \%$ of strains being MDR. A MDR of $91.67 \%$ was observed with 6 resistance profiles for Streptococcus agalactiae strains (Table 3). 
Table 3. Observed resistance profiles for Gram-positive bacteria.

\begin{tabular}{|c|c|c|c|c|c|c|c|c|}
\hline \multicolumn{9}{|c|}{ Resistance patterns observed for strains of Staphylococcus epidermidis } \\
\hline 1 & 2 & 3 & 4 & 5 & $\mathbf{n}$ & $\%$ & OBSER & ATION \\
\hline $\mathrm{AML}^{\mathrm{I}}-\mathrm{AMC}^{\mathrm{S}}-\mathrm{P}^{\mathrm{R}}-\mathrm{CTX}^{\mathrm{S}}$ & $\mathrm{GN}^{\mathrm{R}}-\mathrm{NET}^{\mathrm{S}}$ & $\mathrm{TE}^{\mathrm{R}}$ & $E^{R}-M Y^{R}$ & $\mathrm{CIP}^{\mathrm{S}}-\mathrm{NOR}^{\mathrm{S}}$ & 3 & 23.08 & BMR & \\
\hline$A M L^{R}-A M C^{I}-P^{R}-C T X^{R}$ & $\mathrm{GN}^{\mathrm{R}}-\mathrm{NET}^{\mathrm{R}}$ & $\mathrm{TE}^{\mathrm{R}}$ & $E^{R}-M Y^{R}$ & $\mathrm{CIP}^{\mathrm{R}}-\mathrm{NOR}^{\mathrm{R}}$ & 3 & 23.08 & BMR & $92.30 \%$ \\
\hline$A M L^{R}-A M C^{S}-P^{R}-C T X^{R}$ & $\mathrm{GN}^{\mathrm{R}}-\mathrm{NET}^{\mathrm{S}}$ & $\mathrm{TE}^{\mathrm{R}}$ & $E^{R}-M Y^{R}$ & $C P^{\mathrm{R}}-\mathrm{NOR}^{\mathrm{R}}$ & 2 & 15.38 & BMR & \\
\hline$A M L^{R}-A M C^{S}-P^{R}-C T X^{I}$ & $\mathrm{GN}^{\mathrm{I}}-\mathrm{NET}^{\mathrm{S}}$ & $\mathrm{TE}^{\mathrm{R}}$ & $E^{R}-M Y^{R}$ & $C I P^{R}-N^{R} R^{R}$ & 2 & 15.38 & BMR & \\
\hline$A M L^{R}-A M C^{I}-P^{R}-C T X^{R}$ & $\mathrm{GN}^{\mathrm{R}}-\mathrm{NET}^{\mathrm{S}}$ & $\mathrm{TE}^{\mathrm{R}}$ & $E^{R}-M Y^{I}$ & $C I P^{R}-N^{R} R^{R}$ & 2 & 15.38 & BMR & \\
\hline$A M L^{I}-A^{\prime} C^{S}-P^{R}-C T X^{S}$ & $\mathrm{GN}^{\mathrm{I}}-\mathrm{NET}^{\mathrm{S}}$ & $\mathrm{TE}^{\mathrm{R}}$ & $\mathrm{E}^{\mathrm{I}}-\mathrm{MY}^{\mathrm{S}}$ & $\mathrm{CIP}^{S}-\mathrm{NOR}^{\mathrm{S}}$ & 1 & 7.70 & & \\
\hline \multicolumn{5}{|c|}{ Total } & 13 & 100.00 & & \\
\hline \multicolumn{9}{|c|}{ Resistance patterns observed for strains of Staphylococcus saprophyticus } \\
\hline$A M L^{R}-A M C^{I}-P^{R}-C T X^{R}$ & $\mathrm{GN}^{\mathrm{R}}-\mathrm{NET}^{\mathrm{S}}$ & $\mathrm{TE}^{\mathrm{R}}$ & $\mathrm{E}^{\mathrm{R}}-\mathrm{MY}^{\mathrm{R}}$ & $\mathrm{CIP}^{\mathrm{R}}-\mathrm{NOR}^{\mathrm{R}}$ & 5 & 17.87 & BMR & \\
\hline$A M L^{R}-A M C^{R}-P^{R}-C T X^{R}$ & $\mathrm{GN}^{\mathrm{R}}-\mathrm{NET}^{\mathrm{R}}$ & $\mathrm{TE}^{\mathrm{R}}$ & $\mathrm{E}^{\mathrm{R}}-\mathrm{MY}^{\mathrm{R}}$ & $\mathrm{CIP}^{\mathrm{R}}-\mathrm{NOR}^{\mathrm{R}}$ & 4 & 14.29 & BMR & \\
\hline$A M L^{R}-A M C^{R}-P^{R}-C T X^{R}$ & $\mathrm{GN}^{\mathrm{R}}-\mathrm{NET}^{\mathrm{S}}$ & $\mathrm{TE}^{\mathrm{R}}$ & $E^{R}-M Y^{R}$ & $\mathrm{CIP}^{\mathrm{R}}-\mathrm{NOR}^{\mathrm{R}}$ & 3 & 10.71 & BMR & \\
\hline$A M L^{R}-A M C^{I}-P^{R}-C T X^{R}$ & $\mathrm{GN}^{\mathrm{R}}-\mathrm{NET}^{\mathrm{S}}$ & $\mathrm{TE}^{\mathrm{R}}$ & $\mathrm{E}^{\mathrm{R}}-\mathrm{MY}^{\mathrm{R}}$ & $\mathrm{CIP}^{\mathrm{R}}-\mathrm{NOR}^{\mathrm{R}}$ & 3 & 10.71 & BMR & $85.71 \%$ \\
\hline$A M L^{R}-A M C^{I}-P^{R}-C T X^{R}$ & $\mathrm{GN}^{\mathrm{R}}-\mathrm{NET}^{\mathrm{S}}$ & $\mathrm{TE}^{\mathrm{I}}$ & $E^{R}-M Y^{R}$ & $\mathrm{CIP}^{\mathrm{R}}-\mathrm{NOR}^{\mathrm{R}}$ & 3 & 10.71 & BMR & \\
\hline $\mathrm{AML}^{\mathrm{I}}-\mathrm{AMC}^{\mathrm{S}}-\mathrm{P}^{\mathrm{I}}-\mathrm{CTX}^{\mathrm{R}}$ & $\mathrm{GN}^{\mathrm{S}}-\mathrm{NET}^{\mathrm{S}}$ & $\mathrm{TE}^{\mathrm{R}}$ & $E^{R}-M Y^{R}$ & $C I P^{R}-N^{R}$ & 3 & 10.71 & BMR & \\
\hline$A M L^{R}-A M C^{R}-P^{R}-C T X^{S}$ & $\mathrm{GN}^{\mathrm{S}}-\mathrm{NET}^{\mathrm{S}}$ & $\mathrm{TE}^{\mathrm{R}}$ & $\mathrm{E}^{\mathrm{S}}-\mathrm{MY}^{\mathrm{I}}$ & $\mathrm{CIP}^{\mathrm{S}}-\mathrm{NOR}^{\mathrm{S}}$ & 2 & 7.14 & BMR & \\
\hline$A M L^{I}-A C^{R}-P^{I}-C T X^{I}$ & $\mathrm{GN}^{\mathrm{I}}-\mathrm{NET}^{\mathrm{S}}$ & $\mathrm{TE}^{\mathrm{s}}$ & $E^{I}-M Y^{R}$ & $\mathrm{CIP}^{\mathrm{I}}-\mathrm{NOR}^{\mathrm{R}}$ & 1 & 3.57 & BMR & \\
\hline$A M L^{I}-A C^{R}-P^{R}-C T X^{R}$ & $\mathrm{GN}^{\mathrm{S}}-\mathrm{NET}^{\mathrm{S}}$ & $\mathrm{TE}^{\mathrm{S}}$ & $E^{I}-M Y^{R}$ & $\mathrm{CIP}^{\mathrm{S}}-\mathrm{NOR}^{\mathrm{S}}$ & 4 & 14.29 & & \\
\hline & Total & & & & 28 & 100.00 & & \\
\hline \multicolumn{9}{|c|}{ Resistance patterns observed for Staphylococcus aureus strains } \\
\hline$F O X^{R}-A M L^{R}-A M C^{R}-P^{R}-C T X^{R}$ & $\mathrm{GN}^{\mathrm{R}}-\mathrm{NET}^{\mathrm{S}}$ & $\mathrm{TE}^{\mathrm{R}}$ & $\mathrm{E}^{\mathrm{R}}-\mathrm{MY}^{\mathrm{S}}$ & $C I P^{S}-N^{R}$ & 7 & 24.14 & BMR & \multirow{8}{*}{$89.66 \%$} \\
\hline $\mathrm{FOX}^{\mathrm{R}}-\mathrm{AML}^{\mathrm{R}}-\mathrm{AMC}^{\mathrm{R}}-\mathrm{P}^{\mathrm{R}}-\mathrm{CTX}^{\mathrm{R}}$ & $\mathrm{GN}^{\mathrm{R}}-\mathrm{NET}^{\mathrm{I}}$ & $\mathrm{TE}^{\mathrm{R}}$ & $E^{R}-M Y^{R}$ & $\mathrm{CIP}^{\mathrm{R}}-\mathrm{NOR}^{\mathrm{R}}$ & 5 & 17.25 & BMR & \\
\hline $\mathrm{FOX}^{\mathrm{R}}-\mathrm{AML}^{\mathrm{R}}-\mathrm{AMC}^{\mathrm{R}}-\mathrm{P}^{\mathrm{R}}-\mathrm{CTX}^{\mathrm{R}}$ & $\mathrm{GN}^{\mathrm{R}}-\mathrm{NET}^{\mathrm{S}}$ & $\mathrm{TE}^{\mathrm{R}}$ & $E^{I}-M Y^{R}$ & $\mathrm{CIP}^{\mathrm{R}}-\mathrm{NOR}^{\mathrm{R}}$ & 3 & 10.34 & BMR & \\
\hline$F O X^{R}-A M L^{R}-A M C^{R}-P^{R}-C T X^{R}$ & $\mathrm{GN}^{\mathrm{R}}-\mathrm{NET}^{\mathrm{R}}$ & $\mathrm{TE}^{\mathrm{R}}$ & $E^{R}-M Y^{R}$ & $\mathrm{CIP}^{\mathrm{R}}-\mathrm{NOR}^{\mathrm{R}}$ & 3 & 10.34 & BMR & \\
\hline$F O X^{R}-A M L^{R}-A M C^{R}-P^{R}-C T X^{I}$ & $\mathrm{GN}^{\mathrm{R}}-\mathrm{NET}^{\mathrm{R}}$ & $\mathrm{TE}^{\mathrm{S}}$ & $E^{S}-M Y^{R}$ & $\mathrm{CIP}^{\mathrm{R}}-\mathrm{NOR}^{\mathrm{R}}$ & 3 & 10.34 & BMR & \\
\hline$F O X^{R}-A M L^{R}-A M C^{I}-P^{R}-C T X^{R}$ & $\mathrm{GN}^{\mathrm{I}}-\mathrm{NET}^{\mathrm{S}}$ & $\mathrm{TE}^{\mathrm{R}}$ & $E^{R}-M Y^{R}$ & $\mathrm{CIP}^{\mathrm{R}}-\mathrm{NOR}^{\mathrm{R}}$ & 2 & 6.90 & BMR & \\
\hline$F O X^{S}-A M L^{R}-A M C^{S}-P^{R}-C T X^{S}$ & $\mathrm{GN}^{\mathrm{R}}-\mathrm{NET}^{\mathrm{S}}$ & $\mathrm{TE}^{\mathrm{R}}$ & $E^{R}-M Y^{I}$ & $\mathrm{CIP}^{\mathrm{S}}-\mathrm{NOR}^{\mathrm{S}}$ & 2 & 6.90 & BMR & \\
\hline $\mathrm{FOX}^{\mathrm{I}}-\mathrm{AML}^{\mathrm{R}}-\mathrm{AMC}^{\mathrm{I}}-\mathrm{P}^{\mathrm{R}}-\mathrm{CTX}^{\mathrm{R}}$ & $\mathrm{GN}^{\mathrm{R}}-\mathrm{NET}^{\mathrm{I}}$ & $\mathrm{TE}^{\mathrm{R}}$ & $E^{I}-M Y^{S}$ & $\mathrm{CIP}^{\mathrm{R}}-\mathrm{NOR}^{\mathrm{R}}$ & 1 & 3.45 & BMR & \\
\hline $\mathrm{FOX}^{S}-\mathrm{AML}^{S}-\mathrm{AMC}^{S}-\mathrm{P}^{\mathrm{I}}-\mathrm{CTX}^{S}$ & $\mathrm{GN}^{\mathrm{S}}-\mathrm{NET}^{\mathrm{S}}$ & $\mathrm{TE}^{\mathrm{S}}$ & $E^{S}-M Y^{R}$ & $\mathrm{CIP}^{\mathrm{S}}-\mathrm{NOR}^{\mathrm{S}}$ & 3 & 10.34 & \multicolumn{2}{|c|}{1} \\
\hline \multicolumn{4}{|c|}{ Total } & & 29 & 100.00 & & \\
\hline \multicolumn{9}{|c|}{ Resistance patterns observed for Streptococcus agalactiae strains } \\
\hline$A M P^{R}-A M L^{R}-A M C^{R}-P^{R}-C T X^{R}$ & $\mathrm{GN}^{\star 1}$ & $\mathrm{TE}^{\mathrm{R}}$ & $E^{I}-M Y^{R}$ & $\mathrm{CIP}^{\mathrm{R}}-\mathrm{NOR}^{\mathrm{R}}$ & 7 & 29.17 & BMR & \multirow{5}{*}{$91.67 \%$} \\
\hline$A M P^{R}-A M L^{I}-A M C^{S}-P^{R}-C T X^{S}$ & $\mathrm{GN}^{\star \mathrm{R}}$ & $\mathrm{TE}^{\mathrm{R}}$ & $E^{R}-M Y^{R}$ & $C I P^{R}-N^{R}$ & 5 & 20.83 & BMR & \\
\hline$A M P^{S}-A M L^{I}-A M C^{S}-P^{R}-C T X^{S}$ & $\mathrm{GN}^{\star \mathrm{S}}$ & $\mathrm{TE}^{\mathrm{R}}$ & $E^{R}-M Y^{R}$ & $\mathrm{CIP}^{\mathrm{R}}-\mathrm{NOR}^{\mathrm{R}}$ & 4 & 16.67 & BMR & \\
\hline$A M P^{R}-A M L^{R}-A M C^{I}-P^{R}-C T X^{I}$ & $\mathrm{GN}^{\star S}$ & $\mathrm{TE}^{\mathrm{R}}$ & $E^{R}-M Y^{R}$ & $\mathrm{CIP}^{\mathrm{I}}-\mathrm{NOR}^{\mathrm{R}}$ & 3 & 12.50 & BMR & \\
\hline$A M P^{R}-A M L^{R}-A M C^{I}-P^{R}-C T X^{R}$ & $\mathrm{GN}^{* \mathrm{~S}}$ & $\mathrm{TE}^{\mathrm{R}}$ & $E^{R}-M Y^{S}$ & $\mathrm{CIP}^{\mathrm{S}}-\mathrm{NOR}^{\mathrm{I}}$ & 3 & 12.50 & BMR & \\
\hline \multirow[t]{2}{*}{$A M P^{S}-A M L^{S}-A M C^{S}-P^{R}-C T X^{S}$} & $\mathrm{GN}^{\star \mathrm{S}}$ & $\mathrm{TE}^{\mathrm{R}}$ & $\mathrm{E}^{S}-\mathrm{MY}^{S}$ & $\mathrm{CIP}^{\mathrm{S}}-\mathrm{NOR}^{\mathrm{S}}$ & 2 & 8.33 & \multicolumn{2}{|c|}{ I } \\
\hline & Total & & & & 24 & 100.00 & & \\
\hline
\end{tabular}




\section{Continued}

\begin{tabular}{|c|c|c|c|c|c|c|c|c|}
\hline \multicolumn{9}{|c|}{ Resistance patterns observed for Enterococcus faecalis strains } \\
\hline$A M P^{R}-A M L^{I}-A M C^{S}-P^{R}-C T X^{R}$ & $\mathrm{GN}^{\star \mathrm{R}}$ & $\mathrm{TE}^{\mathrm{R}}$ & $E^{R}-M Y^{R}$ & $\mathrm{CIP}^{\mathrm{R}}-\mathrm{NOR}^{\mathrm{R}}$ & 8 & 30.77 & BMR & \\
\hline$A M P^{S}-A^{A M L} L^{S}-A^{\prime} C^{S}-P^{R}-C T X^{I}$ & $\mathrm{GN}^{* \mathrm{~S}}$ & $\mathrm{TE}^{\mathrm{R}}$ & $E^{R}-M Y^{I}$ & $\mathrm{CIP}^{\mathrm{R}}-\mathrm{NOR}^{\mathrm{R}}$ & 5 & 19.23 & BMR & \\
\hline$A M P^{R}-A M L^{R}-A M C^{S}-P^{R}-C T X^{I}$ & $\mathrm{GN}^{* \mathrm{R}}$ & $\mathrm{TE}^{\mathrm{R}}$ & $E^{R}-M Y^{R}$ & $\mathrm{CIP}^{\mathrm{S}}-\mathrm{NOR}^{\mathrm{S}}$ & 3 & 11.54 & BMR & $73.08 \%$ \\
\hline$A M P^{S}-A M L^{S}-A M C^{S}-P^{R}-C T X^{R}$ & $\mathrm{GN}^{* \mathrm{R}}$ & $\mathrm{TE}^{\mathrm{S}}$ & $E^{R}-M Y^{R}$ & $\mathrm{CIP}^{\mathrm{I}}-\mathrm{NOR}^{\mathrm{I}}$ & 2 & 7.69 & BMR & \\
\hline$A M P^{R}-A M L^{R}-A M C^{R}-P^{R}-C T X^{R}$ & $\mathrm{GN}^{* \mathrm{R}}$ & $\mathrm{TE}^{\mathrm{I}}$ & $E^{I}-M Y^{R}$ & $\mathrm{CIP}^{\mathrm{I}}-\mathrm{NOR}^{\mathrm{R}}$ & 1 & 3.85 & BMR & \\
\hline $\mathrm{AMP}^{\mathrm{s}}-\mathrm{AML}^{\mathrm{s}}-\mathrm{AMC}^{\mathrm{s}}-\mathrm{P}^{\mathrm{I}}-\mathrm{CTX} \mathrm{X}^{\mathrm{s}}$ & $\mathrm{GN}^{* \mathrm{~S}}$ & $\mathrm{TE}^{\mathrm{R}}$ & $E^{S}-M Y^{S}$ & $\mathrm{CIP}^{\mathrm{S}}-\mathrm{NOR}^{\mathrm{S}}$ & 4 & 15.38 & & l \\
\hline \multirow[t]{2}{*}{$A M P^{S}-A^{S} L^{S}-A M C^{S}-P^{I}-C T X^{I}$} & $\mathrm{GN}^{* \mathrm{~S}}$ & $\mathrm{TE}^{\mathrm{I}}$ & $E^{I}-M Y^{R}$ & $\mathrm{CIP}^{\mathrm{S}}-\mathrm{NOR}^{\mathrm{I}}$ & 3 & 11.54 & & I \\
\hline & Total & & & & 26 & 100 & & \\
\hline
\end{tabular}

1: Beta-lactams; 2: Aminoglycosides; 3: Cyclins; 4: Macrolides; 5: Quinolones; BMR: Multi Resistant Bacteria; R: Resistant; S: Sensitive; I: Intermediate.

\section{Resistance of Gram-negative bacteria to the families of antibiotics tested}

Antibiotic resistance patterns observed for Acinetobacter baumannii strains were 7 , and a large proportion of these strains $(76.48 \%)$ were MDR. The strains of Enterobacter cloacae had 7 different antibiotic resistance patterns and were all MDR. The 17 strains of Klebsiella pneumoniae had 7 different antibiotic resistance patterns with MDR of $64.70 \%$. Most of the Pseudomonas aeruginosa strains (82.35\%) were MDR with resistance profiles of 6. The isolated Proteus vulgaris strain showed resistance to all beta-lactam antibiotics and all aminoglycosides tested (Table 4).

\section{Resistance factors of Gram-positive bacteria}

\section{Search for $16 \mathrm{~S}-23 \mathrm{~S}$ genes}

Figure 1 shows the electrophoretic profile of the presence of the $16 \mathrm{~S}-23 \mathrm{~S}$ gene. The search for the $16 \mathrm{~S}-23 \mathrm{~S}$ gene in strains of staphylococci of all species showed that all isolates tested possessed this gene. In Figure 1, the bands observed differed by species and molecular weights ranged from $237 \mathrm{bp}$ to $437 \mathrm{bp}$.

Search for Panton-Valentine Leukocidin in Staphylococcus aureus

None of the Staphylococcus aureus strains isolated produced Panton-Valentine Leucocidin.

\section{Resistance factors of Gram-negative bacteria}

The BLSE phenotype was not detected in any of the Gram-negative bacterial isolates isolated. Of the 4 resistance genes sought (blaTEM, blaSHV, blaCTX-M and blaOXA-1), only the blaTEM and blaSHV genes were found. They were more present in the operating rooms (60\% blaTEM and 50\% blaSHV) than at the other sites. Figure 2 shows the electrophoretic profile of the blaTEM gene. Only the samples in wells $3,5,6$, and 10 possess this gene with a molecular weight of $467 \mathrm{bp}$ in Figure 2.

The electrophoretic profile of the blaSHV gene is shown in Figure 3. In this figure, the blaSHV gene is present in the samples in wells 1 and 6 with a molecular weight of $713 \mathrm{bp}$. 
Table 4. Observed resistance profiles for Gram-negative bacteria.

\begin{tabular}{|c|c|c|c|c|c|c|c|c|}
\hline \multicolumn{9}{|c|}{ Resistance patterns observed for Klebsiella pneumoniae strains } \\
\hline$A M L^{R}-A M C^{R}-P^{R}-C T X^{R}-A T M^{R}-E T P^{R}$ & $\mathrm{NET}^{\mathrm{R}}-\mathrm{GN}^{\mathrm{R}}$ & $\mathrm{TE}^{\mathrm{R}}$ & $\mathrm{CIP}^{\mathrm{I}}-\mathrm{NOR}^{\mathrm{I}}$ & 3 & 17.67 & BMR & & \\
\hline$A M L^{R}-A M C^{R}-P^{R}-C T X^{R}-A T M^{R}-E^{R} P^{R}$ & $\mathrm{NET}^{\mathrm{I}}-\mathrm{GN}^{\mathrm{R}}$ & $\mathrm{TE}^{\mathrm{R}}$ & $C I P^{R}-N^{2} R^{R}$ & 2 & 11.76 & BMR & & \\
\hline$A M L^{R}-A M C^{R}-P^{R}-C T X^{R}-A T M^{R}-E T P^{R}$ & $\mathrm{NET}^{\mathrm{R}}-\mathrm{GN}^{\mathrm{R}}$ & $\mathrm{TE}^{\mathrm{R}}$ & $\mathrm{CIP}^{\mathrm{S}}-\mathrm{NOR}^{\mathrm{I}}$ & 2 & 11.76 & BMR & & $64.70 \%$ \\
\hline$A M L^{R}-A M C^{R}-P^{R}-C T X^{R}-A T M^{R}-E T P^{R}$ & $\mathrm{NET}^{\mathrm{R}}-\mathrm{GN}^{\mathrm{R}}$ & $\mathrm{TE}^{\mathrm{R}}$ & $\mathrm{CIP}^{\mathrm{R}}-\mathrm{NOR}^{\mathrm{I}}$ & 2 & 11.76 & BMR & & \\
\hline$A M L^{R}-A M C^{I}-P^{R}-C T X^{R}-A_{T M}^{R}-E^{R} P^{R}$ & $\mathrm{NET}^{\mathrm{R}}-\mathrm{GN}^{\mathrm{R}}$ & $\mathrm{TE}^{\mathrm{R}}$ & $\mathrm{CIP}^{S}-\mathrm{NOR}^{\mathrm{S}}$ & 2 & 11.76 & BMR & & \\
\hline$A M L^{R}-A M C^{R}-P^{R}-C T X^{R}-A T M^{R}-E T P^{R}$ & $\mathrm{NET}^{\mathrm{I}}-\mathrm{GN}^{\mathrm{S}}$ & $\mathrm{TE}^{\mathrm{I}}$ & $\mathrm{CIP}^{S}-\mathrm{NOR}^{\mathrm{S}}$ & 4 & 23.54 & & 1 & \\
\hline$A M L^{R}-A M C^{R}-P^{R}-C T X^{R}-A T M^{R}-E T P^{R}$ & $\mathrm{NET}^{\mathrm{I}}-\mathrm{GN}^{\mathrm{S}}$ & $\mathrm{TE}^{\mathrm{R}}$ & $\mathrm{CIP}^{\mathrm{S}}-\mathrm{NOR}^{\mathrm{I}}$ & 2 & 11.76 & & l & \\
\hline Total & & & & 17 & 100.00 & & & \\
\hline \multicolumn{9}{|c|}{ Resistance patterns observed for strains of Enterobacter cloacae } \\
\hline$A M P^{R}-A M L^{R}-A M C^{R}-P^{R}-C T X^{R}-A T M^{R}-E T P^{R}$ & $\mathrm{NET}^{\mathrm{R}}-\mathrm{GN}^{\mathrm{R}}$ & $\mathrm{TE}^{\mathrm{R}}$ & $\mathrm{CIP}^{\mathrm{R}}-\mathrm{NOR}^{\mathrm{S}}$ & 5 & 26.32 & BMR & & \\
\hline$A M P^{R}-A M L^{R}-A M C^{R}-P^{R}-C T X^{R}-A T M^{R}-E T P^{S}$ & $\mathrm{NET}^{\mathrm{R}}-\mathrm{GN}^{\mathrm{S}}$ & $\mathrm{TE}^{\mathrm{R}}$ & $\mathrm{CIP}^{\mathrm{I}}-\mathrm{NOR}^{\mathrm{I}}$ & 3 & 15.78 & BMR & & \\
\hline$A M P^{R}-A M L^{I}-A M C^{R}-P^{R}-C T X^{R}-A T M^{R}-E T P^{R}$ & $\mathrm{NET}^{\mathrm{R}}-\mathrm{GN}^{\mathrm{R}}$ & $\mathrm{TE}^{\mathrm{R}}$ & $\mathrm{CIP}^{\mathrm{S}}-\mathrm{NOR}^{\mathrm{S}}$ & 3 & 15.78 & BMR & & \\
\hline$A M P^{R}-A M L^{R}-A M C^{R}-P^{R}-C T X^{R}-A T M^{R}-E T P^{R}$ & $\mathrm{NET}^{\mathrm{R}}-\mathrm{GN}^{\mathrm{R}}$ & $\mathrm{TE}^{\mathrm{R}}$ & $\mathrm{CIP}^{\mathrm{I}}-\mathrm{NOR}^{\mathrm{I}}$ & 2 & 10.53 & BMR & & $100 \%$ \\
\hline$A M P^{R}-A M L^{R}-A M C^{R}-P^{R}-C T X^{R}-A T M^{R}-E T P^{R}$ & $\mathrm{NET}^{\mathrm{R}}-\mathrm{GN}^{\mathrm{R}}$ & $\mathrm{TE}^{\mathrm{S}}$ & $\mathrm{CIP}^{\mathrm{I}}-\mathrm{NOR}^{\mathrm{R}}$ & 2 & 10.53 & BMR & & \\
\hline$A M P^{R}-A M L^{R}-A M C^{R}-P^{R}-C T X^{R}-A T M^{R}-E T P^{R}$ & $\mathrm{NET}^{\mathrm{R}}-\mathrm{GN}^{\mathrm{R}}$ & $\mathrm{TE}^{\mathrm{R}}$ & $\mathrm{CIP}^{\mathrm{S}}-\mathrm{NOR}^{\mathrm{I}}$ & 2 & 10.53 & BMR & & \\
\hline$A M P^{R}-A^{R} L^{R}-A M C^{I}-P^{R}-C T X^{R}-A T M^{I}-E T P^{S}$ & $\mathrm{NET}^{\mathrm{R}}-\mathrm{GN}^{\mathrm{R}}$ & $\mathrm{TE}^{\mathrm{R}}$ & $\mathrm{CIP}^{\mathrm{S}}-\mathrm{NOR}^{\mathrm{S}}$ & 2 & 10.53 & BMR & & \\
\hline Total & & & & 19 & 100.00 & & & \\
\hline \multicolumn{9}{|c|}{ Observed resistance profile for Proteus vulgaris strain } \\
\hline$A M P^{R}-A M L^{R}-A M C^{R}-P^{R}-C T X^{R}-A T M^{R}-E T P^{R}$ & $\mathrm{NET}^{\mathrm{R}}-\mathrm{GN}^{\mathrm{R}}$ & $\mathrm{TE}^{\mathrm{I}}$ & $\mathrm{CIP}^{S}-\mathrm{NOR}^{S}$ & 1 & 100 & & l & \\
\hline Total & & & & 1 & 100.00 & & & \\
\hline \multicolumn{9}{|c|}{ Resistance patterns observed for Acinetobacter baumannii strains } \\
\hline$A M P^{R}-A M L^{R}-A M C^{R}-P^{R}-C T X^{R}-A T M^{R}-E T P^{R}$ & $\mathrm{NET}^{\mathrm{S}}-\mathrm{GN}^{\mathrm{R}}$ & $\mathrm{TE}^{\mathrm{R}}$ & $\mathrm{CIP}^{S}-\mathrm{NOR}^{\mathrm{S}}$ & 4 & 23.54 & BMR & & \\
\hline$A M P^{R}-A M L^{R}-A M C^{R}-P^{R}-C T X^{R}-A T M^{R}-E T P^{R}$ & $\mathrm{NET}^{\mathrm{R}}-\mathrm{GN}^{\mathrm{R}}$ & $\mathrm{TE}^{\mathrm{R}}$ & $C I P^{R}-N^{R}$ & 3 & 17.67 & BMR & & \\
\hline$A M P^{R}-A M L^{R}-A M C^{R}-P^{R}-C T X^{R}-A T M^{R}-E T P^{R}$ & $\mathrm{NET}^{\mathrm{I}}-\mathrm{GN}^{\mathrm{R}}$ & $\mathrm{TE}^{\mathrm{R}}$ & $\mathrm{CIP}^{\mathrm{S}}-\mathrm{NOR}^{\mathrm{I}}$ & 2 & 11.76 & BMR & & $76.48 \%$ \\
\hline$A M P^{R}-A M L^{R}-A M C^{I}-P^{R}-C T X^{R}-A T M^{R}-E T P^{R}$ & $\mathrm{NET}^{\mathrm{R}}-\mathrm{GN}^{\mathrm{R}}$ & $\mathrm{TE}^{\mathrm{R}}$ & $\mathrm{CIP}^{S}-\mathrm{NOR}^{S}$ & 2 & 11.76 & BMR & & \\
\hline$A M P^{R}-A M L^{R}-A M C^{I}-P^{R}-C T X^{R}-A T M^{I}-E T P^{R}$ & $\mathrm{NET}^{\mathrm{S}}-\mathrm{GN}^{\mathrm{R}}$ & $\mathrm{TE}^{\mathrm{S}}$ & $\mathrm{CIP}^{\mathrm{I}}-\mathrm{NOR}^{\mathrm{R}}$ & 2 & 11.76 & BMR & & \\
\hline$A M P^{R}-A M L^{R}-A M C^{R}-P^{R}-C T X^{R}-A T M^{R}-E T P^{I}$ & $\mathrm{NET}^{\mathrm{S}}-\mathrm{GN}^{\mathrm{R}}$ & $\mathrm{TE}^{\mathrm{S}}$ & $\mathrm{CIP}^{\mathrm{S}}-\mathrm{NOR}^{\mathrm{I}}$ & 2 & 11.76 & & l & \\
\hline$A M P^{R}-A M L^{R}-A M C^{R}-P^{R}-C T X^{R}-A T M^{R}-E T P^{I}$ & $\mathrm{NET}^{\mathrm{S}}-\mathrm{GN}^{\mathrm{S}}$ & $\mathrm{TE}^{\mathrm{R}}$ & $\mathrm{CIP}^{\mathrm{S}}-\mathrm{NOR}^{\mathrm{I}}$ & 2 & 11.76 & & 1 & \\
\hline Total & & & & 17 & 100.00 & & & \\
\hline \multicolumn{9}{|c|}{ Resistance patterns observed for Pseudomonas aeruginosa strains } \\
\hline$A M P^{R}-A M L^{R}-A M C^{R}-P^{R}-C T X^{R}-A T M^{R}-E T P^{R}$ & $\mathrm{NET}^{\mathrm{R}}-\mathrm{GN}^{\mathrm{R}}$ & $\mathrm{TE}^{\mathrm{R}}$ & $C I P^{R}-N^{R}$ & 4 & 23.53 & BMR & & \\
\hline$A M P^{R}-A M L^{R}-A M C^{R}-P^{R}-C T X^{R}-A T M^{R}-E T P^{R}$ & $\mathrm{NET}^{\mathrm{S}}-\mathrm{GN}^{\mathrm{R}}$ & $\mathrm{TE}^{\mathrm{R}}$ & $\mathrm{CIP}^{S}-\mathrm{NOR}^{\mathrm{S}}$ & 3 & 17.65 & BMR & & \\
\hline$A M P^{R}-A M L^{R}-A M C^{R}-P^{R}-C T X^{R}-A T M^{R}-E T P^{R}$ & $\mathrm{NET}^{\mathrm{R}}-\mathrm{GN}^{\mathrm{R}}$ & $\mathrm{TE}^{\mathrm{R}}$ & $\mathrm{CIP}^{\mathrm{S}}-\mathrm{NOR}^{\mathrm{I}}$ & 3 & 17.65 & BMR & & $82.35 \%$ \\
\hline$A M P^{R}-A M L^{R}-A M C^{S}-P^{R}-C T X^{R}-A T M^{R}-E T P^{S}$ & $\mathrm{NET}^{\mathrm{R}}-\mathrm{GN}^{\mathrm{R}}$ & $\mathrm{TE}^{\mathrm{R}}$ & $\mathrm{CIP}^{\mathrm{I}}-\mathrm{NOR}^{\mathrm{R}}$ & 2 & 11.76 & BMR & & \\
\hline$A M P^{R}-A M L^{R}-A M C^{R}-P^{R}-C T X^{R}-A^{R} M^{R}-E T P^{S}$ & $\mathrm{NET}^{\mathrm{R}}-\mathrm{GN}^{\mathrm{I}}$ & $\mathrm{TE}^{\mathrm{R}}$ & $\mathrm{CIP}^{\mathrm{S}}-\mathrm{NOR}^{\mathrm{R}}$ & 2 & 11.76 & BMR & & \\
\hline$A M P^{R}-A M L^{R}-A M C^{R}-P^{R}-C T X^{R}-A T M^{R}-E T P^{R}$ & $\mathrm{NET}^{\mathrm{S}}-\mathrm{GN}^{\mathrm{S}}$ & $\mathrm{TE}^{\mathrm{S}}$ & $\mathrm{CIP}^{\mathrm{S}}-\mathrm{NOR}^{\mathrm{I}}$ & 3 & 17.65 & & l & \\
\hline Total & & & & 17 & 100.00 & & & \\
\hline
\end{tabular}

1: Beta-lactams; 2: Aminoglycosides; 3: Cyclins; 4: Quinolones; BMR: Multi Resistant BacteriaR: Resistant; S: Sensitive; I: Intermediate. 


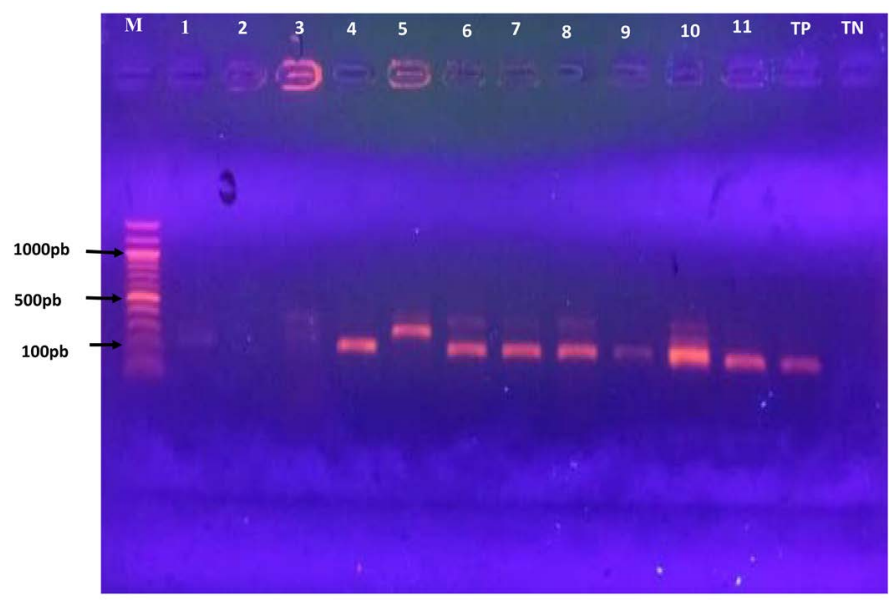

Figure 1. Electrophoretic profile of the presence of the 16S - 23S gene. M: Molecular weight marker; Wells 1 to 11: Positive samples; PT: Positive control; NT: Negative control.

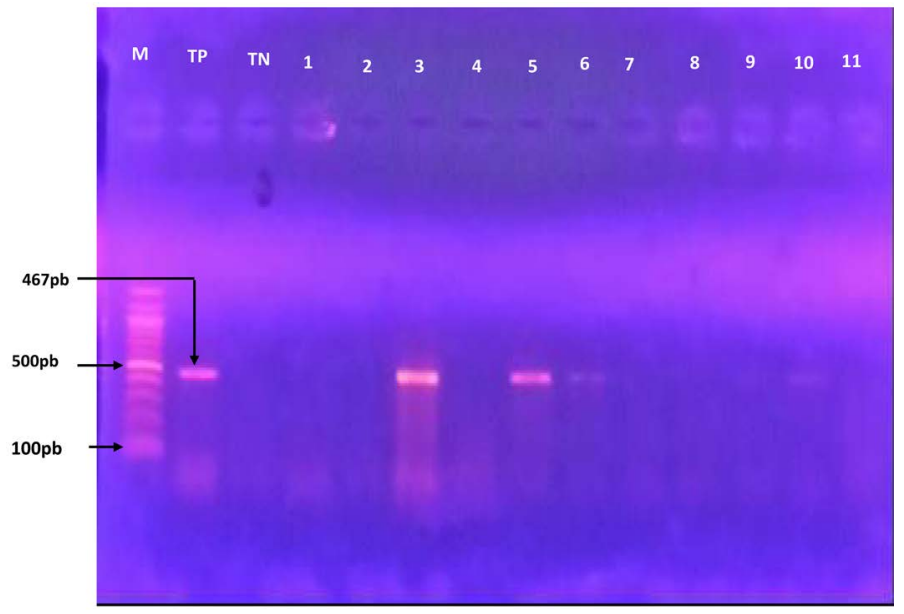

Figure 2. Electrophoretic profile of the presence of the blaTEM gene. Molecular weight marker; PT: Positive control; NT: Negative control; Wells 3, 5, 6 and 10: Positive blaTEM samples; Wells 1, 2, 4, 7, 8, 9 and 11: Negative blaTEM samples.

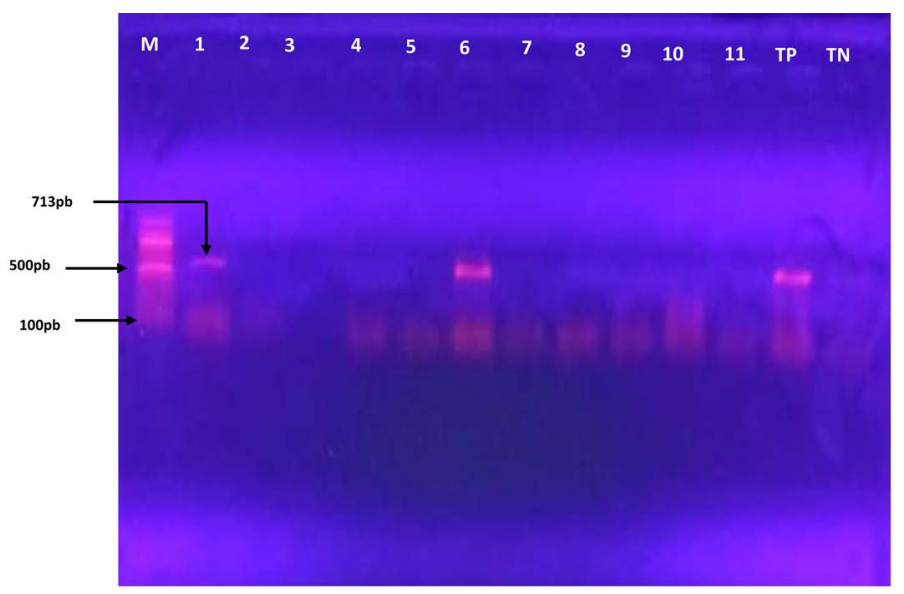

Figure 3. Electrophoretic profile of the presence of the blaSHV gene. M: Molecular weight marker; Wells 1 and 6: Positive blaSHV samples; Wells 2, 3, 4, 5, 7, 8, 9, 10 and 11: Negative blaSHV samples; TP: Positive control; TN: Negative control. 


\section{Discussion}

The results of this study showed that the air, medical-technical equipment, and hands of the staff were contaminated with bacteria. Contamination of the hospital environment is mainly characterized by the interaction between bio-contamination of surfaces, hands, and airborne contamination. This interaction is ensured in the environment by the sedimentation of germs from the air on surfaces, floors, medical-technical equipment, or bedding [19]. The presence of these bacteria in high-risk infectious services could be a risk factor for healthcare-associated infections. It could also result in inadequate compliance with preventive measures by healthcare workers. Coagulase-negative Staphylococcus aureus (29.78\%) and Staphylococcus aureus (17.81\%) were the majority species identified during the study. Their presence could be due to a failure in bio-cleaning and sterilization at CHUZ-SL. These results are comparable to those of Nabila et al., who reported that the bacteria frequently isolated from surfaces at the El Idrissi Hospital in Kenitra, Morocco, are coagulase-negative staphylococci and Staphylococcus aureus [3]. Furthermore, Mora et al. showed that, despite effective cleaning procedures and the use of disinfectants, pathogenic bacteria are generally found on surfaces and other equipment commonly used in hospitals [20].

Taking into account the contamination per department, the predominant bacterial species isolated in neonatology was Staphylococcus aureus (23.91\%). Similar results were obtained by Degbey et al. [21], in the neonatology department of the Departmental and University Hospital Center of Ouémé-Plateau (CHUD-OP), with a rate of $21.95 \%$ of Staphylococcus aureus isolated. Nevertheless, there is a dissimilarity in the other bacterial species isolated in the two neonatology departments. This dissimilarity can be seen in the presence of bacterial species such as Acinetobacter baumannii, Enterobacter cloacae, Enterococcus faecalis, Pseudomonas aeruginosa, and Streptococcus agalactiae in CHUZ-SL and their absence in CHUD-OP. This difference in bacterial species could be due to the composition of the environmental microbiota which differs from one hospital to another, and from one department to another within the same hospital. Considering the presence of pathogens present in the recovery room, the main bacteria isolated were Gram-positive cocci (Enterococcus faecalis, Staphylococcus saprophyticus, and Staphylococcus aureus). Studies conducted on bacterial ecology in intensive care units in Morocco and Cameroon also revealed the presence of various bacteria. The origin of these bacteria may be exogenous, through cross-transmission between patients, staff, and the environment [22] [23]. About to microbiological quality in operating theaters, bacterial contamination was also observed by Gonsu et al. [24] in two reference hospitals in Yaoundé. Degbey et al. [11] in their study conducted at the CNHUHKM identified the species Enterobacter agglomerans versus Enterobacter cloacae at CHUZ-SL. The biodiversity of microorganisms and the composition of the environment could explain this difference in bacterial species.

Based on the results of the antibiogram, it was found that the bacteria isolated 
have variable resistance to the majority of the antibiotics tested. This resistance is also independent of the percentage of bacteria isolated. Indeed, according to Nabila et al. [3] some bacteria, although in small proportion, show a high resistance to most of the antibiotics tested. This is the case in this study of Proteus vulgaris, which had a resistance rate of $66.67 \%$ to the antibiotics tested. The majority of the strains of the 10 bacterial species isolated were MDR bacteria. Indeed, these different strains were resistant to at least three different families of antibiotics. This antibiotic resistance by most of the bacteria isolated was also shown at CNHU-HKM [4] [11]. The existence of a high percentage of MDR bacteria should be of concern to all stakeholders at different levels. These results would explain the potential danger that these bacteria would represent for the health of all patients, health and administrative staff, and visitors to the hospital.

The use of nucleic acid targets, with their high sensitivity and specificity, is an alternative technique for the precise identification and classification of staphylococcal species [25]. The search for the 16S - 23S gene was positive for all strains of staphylococci tested. This genotypic confirmation of the Staphylococcus genus ruled out possible phenotypic identification errors. One of the virulence factors of Staphylococcus aureus is the production of Panton-Valentine Leucocidin. Nevertheless, none of our strains produced this toxin. The strains of Staphylococcus aureus isolated in this study, although pathogenic, are not virulent. The blaCTX-M, blaSHV, blaTEM and blaOXA-1 genes are related to the production of ESBLs by gram-negative bacteria. With respect to the production of $\beta$-extended-spectrum lactamases, the phenotypic detection of their presence was negative for all isolates tested. Amplification of the $\beta$-lactam resistance genes by real-time PCR showed only the presence of the blaTEM and blaSHV genes. The presence of the blaSHV and blaTEM genes shows that they may be present without being phenotypically expressed. Furthermore, the presence of the TEM and SHV genotypes could predict the resistance profile to Cefotaxime (CTX) and Aztreonam (ATM).

The same finding was made by Maina et al., who found in Nairobi, Kenya, that the presence of the SHV gene could predict resistance to Ceftazidime [26]. The coexistence of different resistance genes in gram-negative bacteria may explain their MDR [27] [28]. Therefore, the hospital environment (air, soil, surfaces, equipment) contaminated by MDR bacteria can serve as a reservoir for crosstransmission of these bacteria [29]. This could make therapeutic diagnosis difficult if they were transmitted to humans and pose a public health problem. This study carried out on the characterization of potentially pathogenic bacteria present in the air, on medical-technical equipment, and on the hands of healthcare personnel, could only take into account three services with a high risk of infection. This does not give a global idea of the pathogenic bacteria in all the hospital's departments.

\section{Conclusion}

This study revealed that the microbiological quality of the technical medical 
equipment, the hands of the health personnel, and the air in some high-risk infectious services at the CHUZ-SL are low. We have indeed observed the presence of pathogenic bacteria in these services and MDR in the majority of bacterial strains. Besides, the pathogenicity of these bacteria is strengthened by the acquisition of antibiotic resistance genes (presence of the blaTEM and blaSHV genes). Consequently, prevention measures should be put in place in order to reduce the risk of HCAIs. These preventive measures are going to be focused on the observance of hand hygiene, bio-cleaning, sterilization and disinfection, vaccination of health care personnel, and training in hospital hygiene.

\section{Acknowledgements}

The authors would like to thank all the health professionals of the CHUZ-SL of Cotonou and the staff of the Laboratory of Biology and Molecular Typing in Microbiology for their contribution to the realization of this study.

\section{Authors' Contributions}

C. Degbey and R.O. Quenum are the principal investigators and participated in the planning and carrying out of the study. R.O. Quenum performed data entry and analysis. W. Mousse, A. Sokou, and E. Hounsinou performed the laboratory examinations for the study. H. Sina, L. Baba-Moussa, and H. Bankolé contributed to the writing process. All authors read and approved the final manuscript.

\section{Conflicts of Interest}

The authors declare no conflicts of interest regarding the publication of this paper.

\section{References}

[1] Afle, F.C.D., Quenum, K.J.M., Hessou, S. and Johnson, R.C. (2018) Etat des lieux des infections associées aux soins dans deux hôpitaux publics du sud Benin (Afrique de l'ouest): Centre Hospitalier Universitaire de Zone d'Abomey-Calavi/Sô-Ava et Centre Hospitalier de Zone de Cotonou 5. Journal of Applied Biosciences, 121, 12192-12201. https://doi.org/10.4314/jab.v121i1.9

[2] Hien, H., Drabo, M., Ouedraogo, L., Konfe, S., Sanou, D., Zeba, S., Compaoré, S.C.C., Ouédraogo, J.B. and Méda, N. (2013) Connaissances et pratiques des professionnels de santé sur le risque infectieux associé aux soins: étude dans un hôpital de district au Burkina Faso. Santé Publique, 25, 219-226.

https://doi.org/10.3917/spub.132.0219

[3] Nabila, S., Adil, E., Abedelazi, C., Nabila, A., Samir, H. and Abdelmajid, S. (2014) Rôle de l'environnement hospitalier dans la prévention des infections nosocomiales: Surveillance de la flore des surfaces à l'hôpital El Idrissi de Kenitra-Maroc. European Scientific Journal, 10, 237-247.

[4] Ouendo, E.-M., Dégbey, C., Charles, S.J., Sègnon, J., Saizonou, J. and Makoutodé, M. (2016) Evaluation of the Quality of Medico-Technical Equipment Sterilization in National University Hospital of Cotonou in Benin in 2013. The Open Public Health Journal, 9, 53-64. https://doi.org/10.2174/1874944501609010053

[5] Bloomfield, S.F., Carling, P.C. and Exner, M. (2017) A Unified Framework for Developing Effective Hygiene Procedures for Hands, Environmental Surfaces and 
Laundry in Healthcare, Domestic, Food Handling and Other Settings. GMS Hygiene and Infection Control, 12, 1-16.

[6] Afle, F.C.D., Agbankpe, A.J., Johnson, R.C., Houngbegnon, O., Houssou, S.C. and Bankole, H.S. (2018) Hospital Acquired Infection: Bacteriological Profile of Species from Environmental Surfaces of Cotonou 5 Hospital in South Benin (West Africa). International Journal of Current Microbiology and Applied Sciences, 7, 1503-1515. https://doi.org/10.20546/ijcmas.2018.704.169

[7] Socohou, A., Sina, H., Degbey, C.C., Ahouandjinou, H., Gounou-Kora, H., Baba-Moussa, F., Adjanohoun, A. and Baba-Moussa, L. (2019) Risk Factors and Microbiological Control of Soils, Surfaces and Medical Technical Equipment at the Abomey-Calavi/So-Ava University Hospital Center, Benin. International Journal of Pathogen Research, 3, 1-9. https://doi.org/10.9734/ijpr/2019/v3i130086

[8] Bagheri Nejad, S., Allegranzi, B., Syed, S.B., Ellis, B. and Pittet, D. (2011) HealthCare-Associated Infection in Africa: A Systematic Review. Bulletin of the World Health Organization, 89, 757-765. https://doi.org/10.2471/BLT.11.088179

[9] Ahoyo, T.A., Bankole, H.S., Adeoti, F.M., Gbohou, A.A., Assavedo, S., Amoussou-Guenou, M., Kindé-Gazard, D.A. and Pittet, D. (2014) Prevalence of Nosocomial Infections and Anti-Infective Therapy in Benin: Results of the First Nationwide Survey in 2012. Antimicrobial Resistance and Infection Control, 3, Article No. 17. https://doi.org/10.1186/2047-2994-3-17

[10] Ministère de la santé du Bénin (2006) Décret $N^{\circ} 2006-087$ du 08 mars 2006 portant approbation du document de politique nationale d'hygiène hospitalière en République du Bénin. Journal Officiel de la République du Bénin, 1-14.

[11] Degbey, C., Aguemon, B., Ouendo, E.M., Makoutode, M. and Simon, A. (2013) Etude de la qualité du matériel médico technique utilisé dans les blocs opératoires en vue de la prévention des infections associées aux soins et services au Centre $\mathrm{Na}$ tional Hospitalier et Universitaire de Cotonou-BENIN. Journal de la Société de Biologie Clinique, 18, 29-35.

[12] Denis, F., Ploy, M.-C., Martin, C., Bingen, E. and Quentin, R. (2011) Bactériologie médicale: Techniques usuelles. Masson. (In French)

[13] Boyle-Vavra, S. and Daum, R.S. (2007) Community Acquired Methicillin Resistant Staphylococcus aureus. The Role of Panton-Valentine Leukocidin. Laboratory Investigation, 87, 3-9. https://doi.org/10.1038/labinvest.3700501

[14] Attien, P., Sina, H., Moussaoui, W., Dadié, T., Chabi Sika, K., Djeni, T., Bankole, H.S., Kotchoni, S.O., Edoh, V., Prévost, G., Djè, M. and Baba-Moussa, L. (2013) Prevalence and Antibiotic Resistance of Staphylococcus Strains Isolated from Meat Products Sold in Abidjan Streets (Ivory Coast). African Journal of Microbiology Research, 7, 3285-3293. https://doi.org/10.5897/AJMR2013.5688

[15] Garrec, H., Drieux-Rouzet, L., Golmard, J.-L., Jarlier, V. and Robert, J. (2011) Comparison of Nine Phenotypic Methods for Detection of Extended-Spectrum Beta-Lactamase Production by Enterobacteriaceae. Journal of Clinical Microbiology, 49, 1048-1057. https://doi.org/10.1128/JCM.02130-10

[16] Comité de l'antibiogramme de la Société Française de Microbiologie/European Society of Clinical Microbiology and Infectious Diseases Recommandations. (2018).

[17] Rasmussen, R.S. and Morrissey, M.T. (2008) DNA Based Methods for the Identification of Commercial Fish and Seafood Species. Comprehensive Reviews in Food Science and Food Safety, 7, 280-295. https://doi.org/10.1111/j.1541-4337.2008.00046.x

[18] Dallenne, C., Da Costa, A., Decre, D., Favier, C. and Arlet, G. (2010) Development of a Set of Multiplex PCR Assays for the Detection of Genes Encoding Important 
$\beta$-Lactamases in Enterobacteriaceae. Journal of Antimicrobial Chemotherapy, 65, 490-495. https://doi.org/10.1093/jac/dkp498

[19] Degbey, C.C., Yansambou, M.S., Sopoh, G., Amadou, M.H. and Ouendo, E.M. (2019) Assessment of the Quality of Hospital Hygiene at Gaweye District Hospital in Niamey in 2018. International Journal of Biological and Chemical Sciences, 13, 2233-2244. https://doi.org/10.4314/ijbcs.v13i4.28

[20] Mora, M., Mahnert, A., Koskinen, K., Pausan, M.R, Oberauner-Wappis, L., Krause, R., Perras, A.K., Gorkiewicz, G. and Moissl-Eichinger, C. (2016) Microorganisms in Confined Habitats: Microbial Monitoring and Control of Intensive Care Units, Operating Rooms, Cleanrooms and the International Space Station. Frontiers in $\mathrm{Mi}$ crobiology, 7, 1573-1593. https://doi.org/10.3389/fmicb.2016.01573

[21] Degbey, C., Challa, A., Todedji, J., Koudjo, C., Dossou, A. and Makoutode, M. (2018) Assessment of the Microbiological Quality of the Medico-Technical Equipment, Surfaces, Premises and Staff Hands in the Department of Neonatology of the Departmental University Hospital Center of Oueme-Plateau in Benin in 2018. Journal of Microbiology and Modern Techniques, 4, 104.

[22] Njall, C., Adiogo, D., Bita, A., Ateba, N., Sume, G., Kollo, B., Binam, F. and Tchoua., R. (2013) Écologie bactérienne de l'infection nosocomiale au service de réanimation de l'hôpital Laquintinie de Douala, Cameroun. The Pan African Medical Journal, 14, 140. https://doi.org/10.11604/pamj.2013.14.140.1818

[23] Mortaji, A. (2019) Ecologie bactérienne en réanimation et profil de résistance aux antibiotiques. Thèse de doctorat en médecine, Université Cadi Ayyad, Faculté de médecine et de pharmacie Marrakech.

[24] Gonsu, K.H., Guenou, E., Toukam, M., Ndze, V.N., Mbakop, C.D., Tankeu, D.N., Mbopi-Keou, F.X. and Takongmo, S. (2015) Bacteriological Assessment of the Hospital Environment in Two Referral Hospitals in Yaoundé-Cameroon. The Pan African Medical Journal, 20, Article 224.

https://doi.org/10.11604/pamj.2015.20.224.4433

[25] Sudagidan, M., Yenidunya, A.F. and Gunes, H. (2005) Identification of Staphylococci by 16 S Internal Transcribed Spacer rRNA Gene Restriction Fragment Length Polymorphism. Journal of Medical Microbiology, 54, 823-826.

https://doi.org/10.1099/jmm.0.45868-0

[26] Maina, D., Revathi, G., Kariuki, S. and Ozwara, H. (2012) Genotypes and Cephalosporin Susceptibility in Extended-Spectrum Beta-Lactamase Producing Enterobacteriaceae in the Community. The Journal of Infection in Developing Countries, 6, 470-477. https://doi.org/10.3855/jidc.1456

[27] Anago, E., Ayi-Fanou, L., Akpovi, C.D., Hounkpe, W.B., Tchibozo, M., Bankole, H.S. and Sanni, A. (2015) Antibiotic Resistance and Genotype of Beta-Lactamase Producing Escherichia coli in Nosocomial Infections in Cotonou, Benin. Annals of Clinical Microbiology and Antimicrobials, 14, Article No. 5. https://doi.org/10.1186/s12941-014-0061-1

[28] Koudokpon, H., Dougnon, V., Hadjadj, L., Kissira, I., Fanou, B., Loko, F., Bankole, H.S., Diene, S. and Rolain, J.-M. (2018) First Sequence Analysis of Genes Mediating Extended Spectrum Beta-Lactamase (ESBL) Bla-TEM, $S H V$ - and $C T X$ - $M$ Production in Isolates of Enterobacteriaceae in Southern Benin. International Journal of Infection, 5, 83194-83201. https://doi.org/10.5812/iji.83194

[29] Mousse, W., Sina, H., Mama-Sirou, I., Anago, E., Dah-Nouvlessounon, D., N'Tcha, C., et al. (2019) Antibiotic Resistance and Production of Extended Spectrum $\beta$-Lactamases by Clinical Gram-Negative Bacteria in Benin. Advances in Microbiology, 18, 1-13. https://doi.org/10.9734/jamb/2019/v18i230158 